\title{
Resección laparoscópica de feocromocitoma y quiste pancreático en un paciente con enfermedad de Von Hippel-Lindau
}

\author{
Castillo $\mathrm{O}^{1,2}$, Kerkebe $\mathrm{M}^{1}$, Vitagliano $\mathrm{G}^{1}$, Arellano $\mathrm{L}^{3}$. \\ ${ }^{1}$ Unidad de Endourología y Laparoscopia Urológica Clínica Santa María. ${ }^{2}$ Departamento de Urología, \\ Facultad de Medicina, Universidad de Chile. ${ }^{3}$ Departamento de Anatomía Patológica, Facultad de Medicina, \\ Universidad de Chile.
}

Actas Urol Esp 2007;31(3):292-294

\section{RESUMEN}

\section{RESECCIÓN LAPAROSCÓPICA DE FEOCROMOCITOMA Y QUISTE PANCREÁTICO EN UN PACIENTE CON ENFERMEDAD DE VON HIPPEL-LINDAU}

Introducción: La enfermedad de Von Hippel-Lindau es una condición autosómica dominante, caracterizada por la presencia de hemangioblastomas cerebelosos, angiomas retinianos, quistes y tumores viscerales. Reportamos una experiencia en la resección por vía laparoscópica de un feocromocitoma y quiste pancreático en un paciente con enfermedad de Von Hippel-Lindau.

Material y método: Paciente de 20 años de edad, sexo masculino, con historia de enfermedad de Von HippelLindau, a quien en estudio de hipertensión arterial se le encuentra una masa suprarrenal izquierda de $5 \mathrm{~cm}$. de diámetro y la evaluación bioquímica orienta hacia un feocromocitoma. Es llevado a adrenalectomía laparoscópica y como hallazgo intraoperatorio se encuentra un quiste de $3 \mathrm{~cm}$. en relación a la cola del páncreas, el cual se reseca en el curso de la misma intervención.

Resultados: El tiempo operatorio fue de 120 minutos. No se registraron complicaciones intra o postoperatorias. El sangrado quirúrgico fue $<50 \mathrm{~mL}$ y la estadía hospitalaria fue de 3 días. El estudio de anatomía patológica confirmó el diagnóstico de feocromocitoma de la glándula suprarrenal y la lesión quística fue informada como cistoadenoma microquístico mucoso del páncreas.

Conclusión: La técnica laparoscópica nos ha permitido realizar el abordaje quirúrgico de lesiones en diferentes órganos abdominales en el mismo tiempo operatorio con excelente resultado. A nuestro entender, este es el primer reporte de una adrenalectomía y quistectomía pancreática laparoscópica simultánea.

Palabras clave: Feocromocitoma. Laparoscopia. Quiste pancreático.

\section{ABSTRACT \\ SINGLE-STAGE LAPAROSCOPIC ADRENALECTOMY AND PANCREATIC CYST EXSICION IN A PATIENT WITH VON HIPPEL-LINDAU DISEASE}

Introduction: Von Hippel-Lindau disease is a dominant autosomic hereditary condition, characterized by cerebellar hemangioblastomas, retinal animas and visceral cysts and tumors. We report a case of a patient with Von Hippel-Lindau in which we performed a single-stage laparoscopic adrenalectomy for a pheochromocytoma and pancreatic cyst excision.

Patient and method: A 20 year old male patient with Von Hippel Lindau disease underwent laparoscopic adrenalectomy for a $5 \mathrm{~cm}$ left adrenal mass. A $3 \mathrm{~cm}$ cystic lesion was found of the tail of the pancreas and was resected completely laparoscopically during the same operative procedure.

Results: Total operative time was 120 minutes. There were no operative or postoperative complications. Blood loss was $<50 \mathrm{~mL}$ and hospital stay was 3 days. The histopathologic result was adrenal pheochromocytoma and pancreatic mucous microcystic cystoadenoma.

Conclusion: Laparoscopy allows surgical approach of patients with simultaneous lesions in several abdominal solid viscera, like Von Hippel Lindau disease. This case represents the first report of one-stage laparoscopic adrenalectomy and pancreatic cyst excision.

Keywords: Laparoscopy. Pancreatic cyst. Pheochromocytoma. 
$\mathrm{L}^{\mathrm{a}}$ a enfermedad de Von Hippel-Lindau (VHL) es un síndrome neurocutáneo, de carácter hereditario autosómico dominante, caracterizado por hemangioblastomas cerebelosos y retinianos, quistes y tumores viscerales. La incidencia es de 1 en 36.000 y se presenta con más frecuencia entre la segunda y la tercera décadas de la vida $^{1,2}$.

La lesión más frecuente es el hemangioblastoma cerebeloso, presente en el 83\% de los pacientes. Los hemangiomas retinianos aparecen en cerca de la mitad de los casos. En cuanto a las lesiones quísticas viscerales, las más frecuentes son las pancreáticas (72\%), seguidas por las renales (59\%) y hepáticas (17\%). El carcinoma de células renales aparece en el 51\% de los pacientes y el feocromocitoma en un 7 a $18 \%$, aunque dentro de una línea familiar puede manifestarse hasta en un $57 \%$ de los integrantes ${ }^{1}$.

Los criterios diagnósticos para la enfermedad de VHL son:

1. Más de un hemangioblastoma en el sistema nervioso central (SNC)

2. Un hemangioblastoma en el $\mathrm{SNC}+$ quistes viscerales o carcinoma renal; y

3. Cualquier manifestación con historia familiar $^{1}$.

La laparoscopia ha sido ampliamente utilizada como técnica para el abordaje quirúrgico de los feocromocitomas, estando descritas resecciones totales y parciales para lesiones unilaterales o bilaterales $^{3-5}$ La resección de quistes pancreáticos por vía laparoscópica también ha sido informada, con excelentes resultados quirúrgicos ${ }^{6}$.

Nosotros previamente publicamos nuestra experiencia en cirugía suprarrenal laparoscópica $^{3,7-8}$ y ahora reportamos la resección laparoscópica de un feocromocitoma y quiste pancreático en un paciente con enfermedad de Von HippelLindau.

\section{CASO CLÍNICO}

Paciente de 20 años de edad, sexo masculino, a quien en estudio de hipertensión arterial se le encontró una masa suprarrenal izquierda de 5 cm. de diámetro (Fig. 1) y la evaluación bioquímica mostró catecolaminas plasmáticas y metanefrinas urinarias elevadas. Dentro de sus antecedentes se destaca un hemangioblastoma cere-

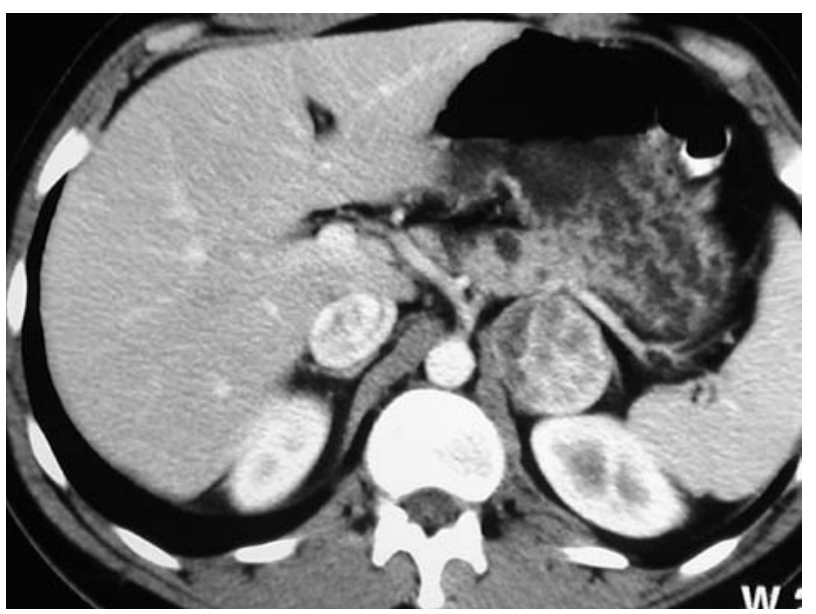

FIGURA 1. Tomografia computada de abdomen muestra masa de $5 \mathrm{~cm}$. en área suprarrenal izquierda en paciente con VHL.

beloso operado dos años atrás, y el antecedente materno de hemangioma cerebeloso intervenido en dos oportunidades.

$\mathrm{Su}$ examen físico general y el examen de fondo de ojo fueron normales.

Fue llevado a adrenalectomía laparoscópica y como hallazgo intraoperatorio se encontró una lesión quística de $3 \mathrm{~cm}$. en relación a la cola del páncreas, la cual se resecó en el curso de la misma intervención (Fig. 2).

El tiempo operatorio fue de 120 minutos, el sangrado fue $<50 \mathrm{~mL}$. No se registraron variaciones significativas de presión arterial durante la cirugía y no hubo complicaciones intra o postoperatorias. La evolución postoperatoria fue satisfactoria, permitiendo que el paciente fuese dado de alta a los tres días de operado.

El estudio de anatomía patológica confirmó el diagnóstico de feocromocitoma de la glándula suprarrenal y la lesión quística fue informada como cistoadenoma microquístico mucoso del páncreas.

\section{DISCUSIÓN}

La enfermedad de VHL representa un desafío médico no sólo por la multicentricidad de las lesiones que la caracterizan sino también por presentarse en niños y adultos jóvenes. La alta incidencia de lesiones quísticas y sólidas viscerales, y la posibilidad de desarrollar nuevas a futuro, hacen de la cirugía laparoscópica un abordaje ideal debido a su baja morbilidad y 


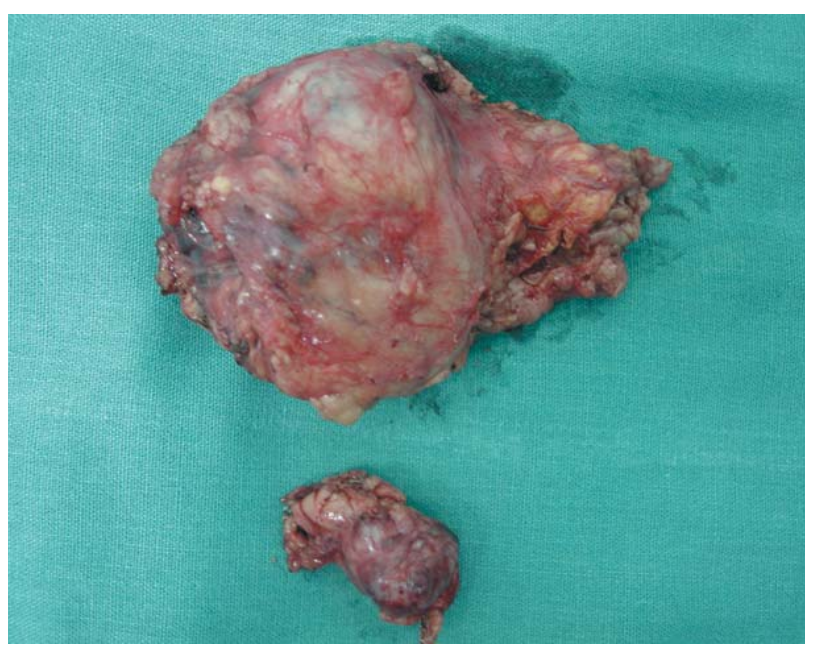

FIGURA 2. Foto macroscópica de Feocromocitoma de la glándula suprarrenal izquierda y Quiste de la cola del páncreas resecado por laparoscopia en paciente con VHL.

a sus excelentes resultados quirúrgicos y cosméticos.

La adrenalectomía laparoscópica para feocromocitomas hereditarios ha mostrado óptimos resultados. $^{4,5}$. La adrenalectomía parcial laparoscópica ha sido un procedimiento reproducido exitosamente en varios centros, siendo de gran utilidad en los casos de tumores bilaterales, frecuentes en la enfermedad de VHL ${ }^{5,9}$.

La incidencia de quistes pancreáticos en VHL supera el 70\%, siendo la manifestación abdominal más frecuente, pero a su vez, la que genera menor sintomatología. Por lo general, los quistes pancreáticos son detectados durante el estudio de otras lesiones como hipernefroma y feocromocitoma $^{1}$.Clinicamente se hacen evidentes por la compresión de los conductos biliares.

La forma de presentación más frecuente de los quistes pancreáticos es el cistoadenoma microquístico, de tamaño y ubicación intra pancreática variable. El cistoadenoma microquístico mucinoso es considerado una lesión pancreática premaligna, pero generalmente no se asocia a VHL. Los quistes y cistoadenomas pancreáticos en esta enfermedad son benignos y solo en casos seleccionados es necesario resecarlos. En este paciente se optó por la resección, debido a que fue un hallazgo intra operatorio y la lesión se encontraba en la cola del páncreas, contiguo al feocromocitoma.
Las resecciones parciales de páncreas por laparoscopia han demostrado ser de utilidad sobretodo en casos de lesiones quísticas y en aquellas localizadas en la cola del páncreas ${ }^{6,10}$.

A nuestro entender, este es el primer reporte de una adrenalectomía y quistectomía pancreática laparoscópica simultánea.

\section{CONCLUSIONES}

La laparoscopia ha demostrado ser una técnica exitosa y de baja morbilidad en el tratamiento quirúrgico de feocromocitomas unilaterales o bilaterales en pacientes con VHL. Creemos que otra de las bondades de esta técnica radica en el hecho de permitir resecar lesiones en otros órganos abdominales en el mismo acto quirúrgico.

\section{REFERENCIAS}

1. Choyke PL, Glenn GM, Walther MM, Patronas MJ, LInehan WM, Zbar B. von Hippel-Lindau disease: genetic, clinical, and imaging features. Radiology. 1995;194(3):629-642.

2. Neumann HP, Zbar B. Renal cysts, renal cancer and von HippelLindau disease. Kidney Int. 1997;51(1):16-26.

3. Lam J., Castillo O, Bravo J, Henriquez R., Tagle F. Feocromocitoma bilateral: Cirugía laparoscópica en 2 casos. Rev Med Chile. 2001;129(1):91-94.

4. Mirallie E, Leclair MD, de Lagausie P, Weil D, Plattner V, Duverne C, et al. Laparoscopic adrenalectomy in children. Surg Endosc. 2001;15(2):156-160

5. Walther MM, Herring J, Choyke PL, Linehan WM. Laparoscopic partial adrenalectomy in patients with hereditary forms of pheochromocytoma. J Urol. 2000;164(1):14-17.

6. Clark GJ, Onders RP, Knudson JD. Laparoscopic distal pancreatectomy procedures in a rural hospital. AORN J. 1997;65(2):334, 337-343.

7. Castillo O, Rossi R, Amat J, van Cauwelaert R, Poblete P, Rodriguez F, et al. Adrenalectomía Laparoscópica: Resultados de una experiencia chilena inicial. Rev Méd Chile. 1999;127(3):304308

8. Castillo OA, Litvak JP, Kerkebe M, Urena RD. Laparoscopic management of symptomatic and large adrenal cysts. J Urol. 2005; 173(3):915-917.

9. Radmayr C, Neumann H, Bartsch G, Elsner R, Janetschek G. Laparoscopic partial adrenalectomy for bilateral pheochromocytomas in a boy with von Hippel-Lindau disease. Eur Urol. 2000; 38(3):344-348.

10. Charukhchyan SA, Lucas GW. Lesser sac endoscopy and laparoscopy in pancreatic carcinoma definitive diagnosis, staging and palliation. Am Surg. 1998;64(9):809-14; discussion 814-816.

Correspondencia autor: Dr. Octavio Castillo

Unidad de Endourología y Laparoscopia Urológica.

Clínica Santa María. Santiago, Chile.

E-mail autor: octaviocastillo@vtr.net

Información artículo: Nota Clínica

Trabajo recibido: noviembre 2006

Trabajo aceptado: enero 2007 